University of Wollongong

Research Online

Faculty of Engineering and Information

Faculty of Engineering and Information

Sciences - Papers: Part A

2017

Processing, characterisation and electromechanical behaviour of elastomeric multiwall carbon nanotubes-poly (glycerol sebacate) nanocomposites for piezoresistive sensors applications

Yi Yan

University of Wollongong, yy619@uowmail.edu.au

Vitor Sencadas

University of Wollongong, victors@uow.edu.au

Jiangshan Zhang

University of Wollongong, jz822@uowmail.edu.au

Guoqing Zu

University of Wollongong, gz854@uowmail.edu.au

Dongbin Wei

University of Wollongong, dwei@uow.edu.au

See next page for additional authors

Follow this and additional works at: https://ro.uow.edu.au/eispapers

Research Online is the open access institutional repository for the University of Wollongong. For further information contact the UOW Library: research-pubs@uow.edu.au 


\title{
Processing, characterisation and electromechanical behaviour of elastomeric multiwall carbon nanotubes-poly (glycerol sebacate) nanocomposites for piezoresistive sensors applications
}

\author{
Abstract \\ Highly stretchable elastomeric multiwall carbon nanotubes - poly (glycerol sebacate) (MWCNT-PGS) \\ nanocomposites were prepared by mixing conductive multiwall carbon nanotubes (MWCNTs) with PGS \\ prepolymer and curing at $120^{\circ} \mathrm{C}$. The incorporation of conductive filler increases the matrix crosslinking \\ density and mechanical stiffness without loss of the flexibility and elasticity of the polymeric network with \\ low mechanical hysteresis behaviour. The percolation threshold for the electrical conductivity was found \\ to be $\sim 1 \mathrm{wt} \%$ of MWCNTs. The piezoresistive behaviour of the prepared samples shows a negative gauge \\ factor (GF) between -0.5 and -0.8 under uniaxial tensile stress, probably due to geometrical factors, \\ whilst under 3-point bending the calculated GF values were positive and a maximum GF $=42$ was \\ achieved for the samples with $2 \mathrm{wt} \%$ MWCNTs. Overall, the unique property combination between the \\ MWCNTs and the PGS make them potentially suitable for the development of large deformation \\ piezoresistive sensors, especially for biomedical engineering applications, flexible and wearable devices.

\section{Publication Details} \\ Yan, Y., Sencadas, V., Zhang, J., Zu, G., Wei, D. \& Jiang, Z. (2017). Processing, characterisation and \\ electromechanical behaviour of elastomeric multiwall carbon nanotubes-poly (glycerol sebacate) \\ nanocomposites for piezoresistive sensors applications. Composites Science and Technology, 142 \\ 163-170. \\ Authors \\ Yi Yan, Vitor Sencadas, Jiangshan Zhang, Guoqing Zu, Dongbin Wei, and Zhengyi Jiang
}

This journal article is available at Research Online: https://ro.uow.edu.au/eispapers/6660 
Processing, Characterisation and Electromechanical Behaviour of Elastomeric Multiwall Carbon Nanotubes-Poly (glycerol sebacate) Nanocomposites for Piezoresistive Sensors

\section{Applications}

Yi Yan, Vitor Sencadas*, Jiangshan Zhang, Guoqing Zu, Dongbin Wei, Zhengyi Jiang* *Corresponding author. Tel.: +61 42214545.

Email address: jiang@uow.edu.au and victors@uow.edu.au School of Mechanical, Materials and Mechatronic Engineering, University of Wollongong, Wollongong, NSW 2522, Australia

Abstract: Highly stretchable elastomeric multiwall carbon nanotubes - poly (glycerol sebacate) (MWCNT-PGS) nanocomposites were prepared by mixing conductive multiwall carbon nanotubes (MWCNTs) with PGS prepolymer and curing at $120{ }^{\circ} \mathrm{C}$. The incorporation of conductive filler increases the matrix crosslinking density and mechanical stiffness without loss of the flexibility and elasticity of the polymeric network with low mechanical hysteresis behaviour. The percolation threshold for the electrical conductivity was found to be 1 wt.\% of MWCNTs. The piezoresistive behaviour of the prepared samples shows a negative gauge factor (GF) between -0.5 and -0.8 under uniaxial tensile stress, probably due to geometrical factors, whilst under 3-point bending the calculated GF values were positive and a maximum GF = 63 was achieved for the samples with 2 wt.\% MWCNTs. Overall, the unique property combination between the MWCNTs and the PGS make them potentially suitable for the development of large deformation piezoresistive sensors, especially for biomedical engineering applications, flexible and wearable devices. 


\section{Introduction}

Recently, wearable multifunctional human-friendly devices [1, 2] were designed with highly stretchable and flexible electric materials, making it possible to monitor human motions for medical [3], physical [4] and other potential applications [5].

To develop suitable materials for the aforementioned purposes, one strategy is to design and fabricate novel structures or combination with the currently established materials. Khang et al. [6] designed a structure with microscale, periodic and wavy silicon on the rubber substrate. Large tensile and compressive strain could be applied on the structure without much substantial strain on the silicon. Kim et al. [7] fabricated stretchable silicon circuit with wave-like structure on plastic and elastomeric substrates.

Another approach is to utilise different materials to assemble into devices. Different types of materials have been reported for flexible sensors applications. Gullapalli et al. [8] embedded zinc oxide particles into cellulose fibres by a solvothermal method for flexible strain sensor applications. Poly(dimethyl siloxane) (PDMS) is widely used for the fabrication of stretchable strain sensors by adding different conductive fillers, e.g. silver nanowires [9], carbon black particles [10], carbon nanotubes [11] or graphene layers [12], with a maximum strain between 50 up to $280 \%$, and capable to detect and monitor human motion.

Carbon nanotubes (CNTs) have outstanding mechanical, thermal, electrical properties, and are one of the most used fillers not only to improve the mechanical properties but also to induce electrical conductivity to insulator polymeric matrix [13]. CNT dispersion into the polymeric matrix plays an important role in nanocomposites mechanical and electrical behaviour and is one of the most studied topics in order to enhance the overall physical and chemical properties of the materials. Different techniques are used to obtain a homogeneous dispersity of the filler, including treatment with chemical solvents (ethanol, 
xylene, etc.) [14]; mechanical mixing, plasma treatment and ultra-sonication [15], being the last one applied with more success to separate the carbon bundles and reducing cluster size, promoting the better CNT dispersion, reducing the percolation threshold, and consequently achieve an enhanced electrical and mechanical properties of the polymer based nanocomposites $[15,16]$.

Poly (glycerol sebacate) (PGS) is a tough biodegradable elastomer which was first reported by Wang et al. [17]. PGS is synthesised by a two-step poly-condensation of glycerol and sebacic acid, which are inexpensive, in-vivo safe and approved for medical applications by US Food and Drug Administration [18]. Controlling the curing time, temperature and other parameters during synthesis, its mechanical properties and degradation kinetic can be tailored [19]. Nanocomposites MWCNT-PGS were prepared by Gaharwar et al. [20], and it was found that the addition of the filler increases the overall mechanical stiffness without compromise of the elasticity of the polymeric PGS matrix, especially under compressive mechanical load. Nevertheless, no electrical characterization was reported and the electrical conductivity behaviour of those materials is unknown. In this work, MWCNT-PGS composites with different conductive filler concentrations were prepared by solvent casting method. Firstly, PGS pre-polymer was synthesised by a two-step poly-condensation of glycerol and sebacic acid. After, different concentrations of MWCNTs were added to the prepolymer, and cured at $120^{\circ} \mathrm{C}$ for $48 \mathrm{~h}$. The effect of the carbon nanofiller in the polymer morphology was characterised by scanning electron microscopy, and dynamic and quasistatic mechanical properties were evaluated. The electrical properties of the nanocomposites were systematically addressed as well as the electromechanical performance through the calculation of the samples gauge factor (GF). The material fundamental properties were discussed and the relationship between the processing 
method and sample mechanical and electrical properties were discussed, as well as its potential for use in large strain sensor applications.

\section{Experimental}

\subsection{Materials and processing}

MWCNT-PGS composites were synthesised according to the published methods $[17,20]$. Equimolar of glycerol (Sigma-Aldrich Australia) and sebacic acid (Sigma-Aldrich Australia) were mixed at $120^{\circ} \mathrm{C}$ under argon for $24 \mathrm{~h}$ to prepare pre-polymer for further treatment. Pre-polymer was poured into a Teflon mould and cured at $120^{\circ} \mathrm{C}$ for $48 \mathrm{~h}$ under a 100 mTorr vacuum.

Nanocomposite films were prepared by dispersing the desired amount of multiwall carbon nanotubes (Play with carbon, Australia) in tetrahydrofuran (THF, Sigma Australia) solution with the help of an ultrasound bath (Bandelin, Sonorex Super RK106) for $2 \mathrm{~h}$. After dispersion, the pre-polymer was added to the solution and stirred at room temperature until complete dissolution. The relation of pre-polymer to THF was $1 \mathrm{~g}$ to $5.5 \mathrm{~mL}$, respectively. The amount of MWCNT presented in the solution was selected to result in concentration between 0 to 3 wt.\% of filler presented in the PGS matrix. After complete dissolution, the solution was poured into a clean Teflon mould and solvent evaporation was performed overnight at room temperature. Highly flexible films were obtained by curing the pre-polymer in the conditions stated above (vacuum oven at 100 mTorr, for $48 \mathrm{~h}$ ).

\subsection{Sample morphology}

The MWCNT-PGS samples were fractured after immersion into liquid nitrogen for $30 \mathrm{~min}$. The platinum coatings were deposited on the cross-section of the samples by Dynavac 
Sputter Coater with the thickness of around $10 \mathrm{~nm}$. After $24 \mathrm{~h}$ in the desiccator, the images of the cross-section were taken by JEOL 7500 field emission SEM.

\subsection{Mechanical properties}

The PGS and MWCNT-PGS samples were cut into dog bone shape of $2 \mathrm{~mm}$ wide and 18 $\mathrm{mm}$ for gauge length, and a thickness of $1 \mathrm{~mm}$. Quasi-static tensile tests and cyclic tensile tests were performed with a Shimadzu EZ-L mechanical tester (10 N load cell). For single tensile tests, stroke speed was set as $1 \mathrm{~mm} / \mathrm{min}$, at room temperature $\left(\sim 22^{\circ} \mathrm{C}\right)$. Mechanical cyclic experiments were performed in the same experimental conditions as quasi-static test, until a maximum strain of 5, 10 and $20 \%$, for 10 cycles, at a stroke speed of $1 \mathrm{~mm} / \mathrm{min}$.

\subsection{Electrical conductivity}

Sample electrical resistance was calculated from the slope of the I-V curves measured with an automated picoammeter/voltage source (Keithley 487). The I-V data were collected in a $1 \mathrm{~mm}$ thick sample between $6 \mathrm{~mm}$ diameter gold contacts, previously deposited with the Dynavac Sputter Coater. The applied voltage ranged between -20 and $+20 \mathrm{~V}$, and the volume resistivity $(\rho)$ of the sample was calculated through Eq. (1):

$$
\rho=\frac{R A}{t}
$$

where $R, A$ and $t$, are the measured sample electrical resistance, electrode area and sample thickness, respectively. The electrical conductivity $(\sigma)$ was calculated from $\sigma=\frac{1}{\rho}$. At least three measurements were performed for each sample.

\subsection{Electromechanical characterisation}

The samples for sensor application were cut into rectangle shape with the size of $40 \mathrm{~mm}$ $x 8 \mathrm{~mm} \times 1 \mathrm{~mm}$. Electromechanical experiments were performed by measuring the electrical resistance of the sample through metallic contacts placed on the surface of the sample, with 
an Agilent 34410A multimeter during the mechanical measurement of the sample, following the method described elsewhere [14] in a Shimadzu EZ-L (10 N load cell). Two different mechanical solicitations were applied to the samples. In the denominated method 1 , the samples were placed between the clamps and submitted to a tensile load without and with a pre-strain (5, 10 and $20 \%$ deformation). The evaluation of the piezoresistive response during the uniaxial stress tests was performed at different stroke rates $(20,30,40$ and 50 $\mathrm{mm} / \mathrm{min}$ ) and strain levels between 1 to $50 \%$. The influence of the pre-strain was studied by applying a pre-strain of 5, 10 and $20 \%$ to the samples, and the mechanical experiment was performed at a fixed stroke speed of $40 \mathrm{~mm} / \mathrm{min}$. In the method 2, the samples were submitted to 3-point bending measurements, where the samples were fixed to the surface of a polypropylene strip (thickness of $1 \mathrm{~mm}$ ). Gold electrodes with an area of $6 \mathrm{~mm} \times 1 \mathrm{~mm}$ were placed at the surface of the samples and a displacement of $1 \mathrm{~mm}$ was applied to the sample. For all the electromechanical experiments, 10 loading-unloading cycles were performed at room temperature, and the average electromechanical response was evaluated.

Variations of the electrical resistance due to the mechanical load were quantitatively evaluated by the gauge factor (GF), defined as Eq. (2):

$$
G F=\frac{\partial R / R_{0}}{\partial l / l_{0}}
$$

where $R_{0}$ is the sample electrical resistance without mechanical deformation, and $\partial R$ is the resistance change promoted by the change in sample length $\partial l$ from the original length $I_{0}$. GF can also be expressed as Eq. (3):

$$
G F=\frac{\partial R / R_{0}}{\varepsilon_{l}}=\frac{\partial \rho / \rho_{0}}{\varepsilon_{l}}+1+2 v
$$


where $\varepsilon_{l}=\partial l / l_{0}$ (strain), and $v$ is the material Poisson's ratio. Eq. (3) shows that the GF has a contribution from the intrinsic piezoresistivity $\left(\frac{\partial \rho / \rho_{0}}{\varepsilon_{l}}\right)$ and the other from a geometrical effect $(1+2 v)[21]$

For uniaxial tensile experiments (method 1), Eq. (2) was used to evaluate the GF, while for the 3-point bending measurements (method 2), the strain was calculated from the theory of a pure bending of plates, valid between the inner loading points [22], in Eq. (4):

$$
\varepsilon=\frac{6 D d}{L^{2}}
$$

where $D$ is the displacement of the cross-head, $d$ is the thickness of the strip and sample, $\mathrm{L}$ is the distance between the supporting rods.

\section{Results and discussion}

\subsection{Sample morphology and MWCNT distribution}

The characteristics SEM micrographs obtained for the neat PGS and MWCNT-PGS with different amounts of fillers are presented in Figure 1. The cross-section of the PGS is quite smooth without the presence of pores (Figure 1). It was observed that the MWCNTs are randomly distributed among the polymer matrix, and the absence of big clusters was noticed even for higher filler concentrations, which suggests that the dispersion method and the conditions used promote an efficient distribution of the conductive filler among the polymer matrix were suitable.

\section{Figure 1}

\subsection{Mechanical properties}

Representative quasi-static stress-strain curves for the PGS nanocomposites are presented in Figure 2. The PGS samples show a characteristic elastomeric behaviour, and an 
increase of the maximum stress was observed with increasing the amount of MWCNTS presented in the composite samples.

\section{Figure 2}

The improvement of the mechanical properties with the filler content suggests that the filler is probably covalently crosslinked to the PGS polymer chains. In the special case of the elastomeric materials, the crosslinking density $(n)$ is related to the elastic modulus $(E)$ through [23] Eq. (5):

$$
E=3 R T n
$$

where $R$ is the universal gas constant and $T$ is the absolute temperature, and the obtained results are presented in Table 1 . The crosslinking density of pure PGS is $11 \pm 1 \mathrm{~mol} / \mathrm{m}^{3}$, a value in the range reported by Chen et al. [24]. The addition of MWCNTs results in a more than two-fold increase of the cross-linking density for the sample with 1 wt.\% MWCNTs (30 $\pm 1 \mathrm{~mol} / \mathrm{m}^{3}$ ) and more than nine-fold for the sample with $3 \mathrm{wt} . \%$ filler added to the polymer matrix (Table 1). Nevertheless, the increase of filler content above $1 \mathrm{wt} . \%$ in the polymeric matrix results in a decrease in the total elongation of the nanocomposites. Pure PGS shows an ultimate strain of $219 \pm 8 \%$, and similar result was obtained for the sample with 1 wt.\% MWCNTs. The incorporation of higher amounts of MWCNTs reduce the nanocomposites maximum elongation down to $119 \pm 13 \%$ for the sample with 3 wt.\% filler. Such behaviour is expected due to the increase of the crosslinking density with the addition of the filler to PGS matrix.

Gaharwar et al. [20] reported that there is a decrease of the maximum elongation of the nanocomposites samples with the increase of MWCNTs presented in the PGS sample due to the increase of polymer crosslinking density promoted by the filler. However, the 
mechanical properties presented by samples prepared in this work shows, in general and improved behaviour, with similar Young's modulus when compared to samples prepared elsewhere $[20,24]$, but with higher total strain and ultimate stress. It was reported that increasing polymer cure temperature leads to an increase of the $E$ and maximum stress, but the deformation at break decreases, probably due to the increase of crosslinking density of the polymer matrix.

Table 1 - Mechanical properties and degree of crosslinking for PGS and composite samples with different MWCNT amounts.

\begin{tabular}{c|cccc}
\hline $\begin{array}{c}\text { MWCNT } \\
\text { amount }\end{array}$ & Ultimate & Ultimate stress & Young's & Crosslinking \\
(wt.\%) & & & & \\
(kPa) & & & \\
\hline $\mathbf{0}$ & $219 \pm 8$ & $175 \pm 11$ & $88 \pm 9$ & $11 \pm 1$ \\
$\mathbf{1}$ & $233 \pm 25$ & $516 \pm 74$ & $216 \pm 13$ & $30 \pm 1$ \\
$\mathbf{2}$ & $134 \pm 8$ & $645 \pm 51$ & $434 \pm 39$ & $65 \pm 5$ \\
$\mathbf{3}$ & $119 \pm 13$ & $893 \pm 102$ & $764 \pm 44$ & $101 \pm 4$ \\
\hline
\end{tabular}

The PGS nanocomposite samples, with different amounts of MWCNTs, were submitted to cyclic stress-strain experiments at different deformations: 5, 10 and $20 \%$, and the characteristic loading-unloading stress-strain curves for cycle number 10 are presented in Figure 3. Neat PGS matrix stress unloading follows almost the same path of the loading stress, which suggests that for the applied deformation conditions, the polymer chains are flexible enough to change their configuration when stretched (Figure 3a). Such behaviour is characteristic of "perfect" elastomeric materials like $\alpha$-elastin prepared from bovine 
ligamentum nuchae [25]. For nanocomposite samples, it was observed that the mechanical stress during the unloading does not follow the loading curve, and a mechanical energy loss was possible to observe (Figure $3 b$ ).

The effect of the MWCNTs on the energy absorbed by the polymeric network during the mechanical loading-unloading cycles was evaluated (Figure 3c). Pure PGS shows an energy absorption of $0.07 \pm 0.005 \mathrm{~kJ} / \mathrm{m}^{3}$ after the $10^{\text {th }}$ cycle and for a deformation of $5 \%$, while the nanocomposite sample with $3 \mathrm{wt} . \%$ of filler presents an energy loss of $0.176 \pm 0.016 \mathrm{~kJ} / \mathrm{m}^{3}$. The energy loss after the $10^{\text {th }}$ cycle for the different strains applied reveals that for all of the samples, the energy loss increases with the amount of the strain applied, and with the concentration of filler presented in the polymeric matrix (Figure 3d). It was observed in Figure $3 c$ that the sample stiffness increases with the amount of carbon nanotubes added to the PGS, probably due to the load transfer efficiency within the crosslinked network. The increase of the crosslinking density in the PGS nanocomposite samples increases the number of rigid regions presented among the matrix and for the applied cyclic stress conditions, these regions require more energy to follow the mechanical deformation and during the loading-unloading of the mechanical solicitation, there is a loss of energy, probably in form of heat. Nevertheless, the amount of energy loss in any of the samples is lower, which shows the potential of these materials for large strain applications, without losing significantly their mechanical performance.

\section{Figure 3}

\subsection{Electrical and electromechanical behaviour}

MWCNTs-PGS nanocomposites electrical resistance was measured by two-point method. The electrical conductivity of PGS polymer and MWCNT-PGS nanocomposites are presented in Figure 4. It was observed that the conductivity increases with the increase of filler content 
present in the polymer matrix, especially for concentration above $1 \mathrm{wt} . \%$, where, probably a continuous carbon nanotube network is available to promote the electrical conductive path for the electron charges. The results obtained show that the rise of the electrical conductivity occurs in a smooth fashion, rather than in a sharp way as the percolation theory envisages. This behaviour can be attributed to an enhanced distribution of the MWCNTs among the polymer matrix (Figure 1). An inflection point of the conductivity curve can be observed around $1 \mathrm{wt} . \%$, and this is considered to estimate the percolation threshold [26]. The percolation threshold found for these samples is in the range reported for other MWCNT-polymer systems [14].

Nanocomposites with a filler concentration near the percolation threshold, in general have a greater electrical resistance variation and consequently higher gauge factor (GF) [27], but the small amount of conductive filler presented in those samples hinders their use for large strain applications, due to the strong drop of conductivity that arises from the higher deformation, promoted by the destruction of the conductive path, and consequently only deformation as high as $5 \%$ can be applied [28]. For large strain sensors, conductive filler concentration above the percolation threshold are required in order to undergo deformations as higher as $50 \%$, without destroying the conductive path promoted by the carbon nanotubes and a linear variation of the electrical resistance with values compatible with strain sensors can be achieved $[27,28]$.

\section{Figure 4}

The electromechanical performance of the MWCNT-PGS samples with 2 and 3 wt.\% conductive fillers was examined under uniaxial tensile stress and in 3-point bending experiments. Firstly, uniaxial testing was performed at different stroke speeds, without any pre-strain applied to the sample, and the GF evolution was determined by the variation of 
the electrical resistance of the samples during the loading and unloading cycles (Figure 5a and b), according to Eq. (2). For the mechanical conditions tested, it was observed that the MWCNT-PGS samples follow in a linear fashion the applied mechanical solicitation, both during the loading and unloading cycles (Figure 5a and b), which suggests an excellent electromechanical response for the prepared samples. It was also observed that these materials have a small mechanical hysteresis (Figure 3), demonstrating good reliability and stability, making them outstanding candidates for high deformation strain sensors. Further, it is possible to observe that when the material is stretched, the electrical resistance decreases and according to Eq. (2), where the slope of $\frac{R-R_{0}}{R_{0}} \sim \varepsilon$ determines the GF of the samples. A negative slope was obtained for the samples with 2 and 3 wt.\% MWCNTs, which is an unusual characteristic for polymeric nanocomposites based in carbonaceous materials $[14,26,29]$. Negative GF values $(-4.9)$ were found by Lu et al. [5] for the carbon black doped poly (dimethylsiloxane) (PDMS) and such behaviour was attributed to the Poisson's effect, where lateral contraction occurs in conjunction with longitudinal elongation. Graphene was deposited on the surface of pre-stretched PDMS films by Wang et al. [30]. In their work, they reported a GF value of $\sim-2$, and this behaviour was attributed to the buckling effect promoted by the pre-strain applied to the polymer substrate during the graphene transfer. In the case of the MWCNT-PGS samples, the negative GF values found are probably related to the Poisson's ratio effect. When the sample is stretched, the length of the sample increases in the longitudinal direction, but a reduction of thickness and width occurs, leading to a decrease of the perpendicular area. While the distance between the MWCNTS in the stretching direction increases, making more difficult for the electrons to find a conductive path along the filler network, and expecting to lead to an increase of sample electrical resistance. Nevertheless, the decrease of the cross section area, perpendicular to 
the mechanical load, probably promotes new pathways for the electrons to move along the conductive filler, increasing the tunnelling effect and leading to a decrease of the electrical resistance. The overall GF values observed for the MWCNT-PGS samples are probably a result of the competition between this two effects.

The effect of the strain rate on the GF was characterised by submitting the nanocomposite samples, without pre-stretch to different mechanical solicitations, for a total strain of $50 \%$ (Figure $5 \mathrm{c}$ ). It was observed that the GF changes from a value of -0.6 down to -0.8 for the sample with 2 wt.\% MWCNTs, while the sample with higher filler content presents a similar GF value, around -0.8, independently of the stroke speed applied (Figure 5c). The effect of the pre-strain applied to the piezoresistive samples was also evaluated (Figure 5d), and it was observed that the increase of pre-strain leads to an increase of the GF value form -0.8 up to -0.5 , for a $10 \%$ pre-strain, remaining constant for higher predeformation of the sample. It was interesting to observe that both samples (with 2 and $3 \mathrm{wt} . \%$ MWCNTs) present similar trend, which is a suggestion that the negative GF values found for this samples are due to the Poisson's effect.

\section{Figure 5}

The characteristic results obtained for the 3-point bending experiments are presented in Figure 6, where 10 mechanical loading-unloading cycles are shown for the samples with 2 and 3 wt.\% of MWCNTs. The variation of samples electrical resistance follows the mechanical deformation over time. In this method, the applied stress is parallel to the crosssection of the sample, rather than normal as in the case of the method 1 of the uniaxial tensile experiments. In the 3-bending mode there is a non-uniform stress and strain distribution along the cross-section, which is higher at the outer surfaces of the specimen; 
being the surface where the load is applied in a compressive state and in the tensile mode at the opposite surface.

The evolution of the GF obtained for the composite samples with 2 and 3 wt.\% conductive fillers at different stroke speeds is presented in Figure $6 c$. It was observed that the sample with 3 wt.\% MWCNTs presents a GF around 5 for small stroke speed, increasing up to 13 for a stroke rate of $3 \mathrm{~mm} \cdot \mathrm{min}^{-1}$, and decreasing again until a minimum of GF = 5 for higher mechanical strain rates. For the sample with $2 \mathrm{wt}$.\% conductive filler the observed GF values are higher than the ones obtained for the more conductive sample, while the GF evolution trend at the different stroke speeds is similar. Values of GF between 16 and 63 were obtained, being the maximum gauge factor obtained at a $3 \mathrm{~mm} \cdot \mathrm{min}^{-1}$ deformation speed. The difference observed for the GF values between the samples could be explained by percolation threshold theory, where the sample with $2 \mathrm{wt.} \%$ is near the percolation threshold ( 1 wt.\%) of the piezoresistive material (Figure 3 ), and it is known that this is the region where small deformations lead to the highest change in the sample electrical resistance [27], increasing the overall GF of the samples.

It is interesting to observe that the GF values found for the 3 point-bending method are positive, when compared to the negative ones calculated for the samples submitted to the uniaxial stretching deformation (Figure 5). It was reported that the GF values are a contribution of two effects: (1) the intrinsic piezoresistive effect and (2) the geometric variation effect. In elastomeric materials, GF values up to $\sim 2$ are mostly due to the geometrical contributions, as observed for the samples submitted to the uniaxial tensile stress, while the larger GF values are due to electrical resistance variation of the network conductive fillers established in the nanocomposite samples and therefore to intrinsic contributions. 


\section{Figure 6}

\section{Conclusions}

Highly stretchable elastomeric MWCNT-PGS nanocomposites were processed, firstly by synthesising the PGS pre-polymer and after mixing the conductive filler and curing the samples at $120{ }^{\circ} \mathrm{C}$. Nanocomposite materials mechanical and electrical properties are influenced by the amount of conductive filler added to the polymer matrix. The addition of 3 wt.\% MWCNTs results in a nine-fold increase in Young's modulus, accompanied by a $50 \%$ decrease of the strain at break. PGS sample shows the "perfect" elastomeric behaviour under cyclic uniaxial tensile stress, while the amount of energy loss increases with the nanofillers concentration and maximum strain applied. The addition of filler to the polymeric elastomer leads to an increase of the crosslinking density and electrical conductivity, with a percolation threshold around 1 wt.\% MWCNTs concentration, under the given experimental conditions. The piezoresistive behaviour of the prepared samples shows a negative gauge factor $-0.5>\mathrm{GF}>-0.8$ during uniaxial tensile stress, probably due to geometrical factors, whilst under 3 point-bending the GF values found are positive and up to 63 , for the sample near the percolation threshold.

Overall, the unique property combinations between the MWCNTs and the PGS make them potentially suitable for the development of large deformation piezoresistive sensors, especially for biomedical engineering applications.

\section{Acknowledgements}


The authors acknowledge financial support from China Scholarship Council (CSC), UPA from University of Wollongong and Australian Research Council (ARC) for Future Fellowship (Z.Y. Jiang). The authors acknowledge use of facilities within the UOW Electron Microscopy Centre.

\section{References}

[1] Munro BJ, Campbell TE, Wallace GG, Steele JR. The intelligent knee sleeve: A wearable biofeedback device. Sensors and Actuators B: Chemical. 2008;131(2):541-547.

[2] Enzo Pasquale S, Lorussi F, Mazzoldi A, Rossi DD. Strain-sensing fabrics for wearable kinaesthetic-like systems. IEEE Sensors Journal. 2003;3(4):460-467.

[3] Inpil K, Mark JS, Jay HK, Vesselin S, Donglu S. A carbon nanotube strain sensor for structural health monitoring. Smart Materials and Structures. 2006;15(3):737.

[4] Helmer RJN, Farrow D, Ball K, Phillips E, Farouil A, Blanchonette I. A pilot evaluation of an electronic textile for lower limb monitoring and interactive biofeedback. Procedia Engineering. 2011;13:513-518.

[5] Lu N, Lu C, Yang S, Rogers J. Highly Sensitive Skin-Mountable Strain Gauges Based Entirely on Elastomers. Advanced Functional Materials. 2012;22(19):4044-4050.

[6] Khang D-Y, Jiang H, Huang Y, Rogers JA. A Stretchable Form of Single-Crystal Silicon for High-Performance Electronics on Rubber Substrates. Science. 2006;311(5758):208-212. [7] Kim D-H, Ahn J-H, Choi WM, Kim H-S, Kim T-H, Song J, et al. Stretchable and Foldable Silicon Integrated Circuits. Science. 2008;320(5875):507-511.

[8] Gullapalli H, Vemuru VSM, Kumar A, Botello-Mendez A, Vajtai R, Terrones M, et al. Flexible Piezoelectric ZnO-Paper Nanocomposite Strain Sensor. Small. 2010;6(15):16411646. 
[9] Amjadi M, Pichitpajongkit A, Lee S, Ryu S, Park I. Highly Stretchable and Sensitive Strain Sensor Based on Silver Nanowire-Elastomer Nanocomposite. ACS Nano. 2014;8(5):51545163.

[10] Mattmann C, Clemens F, Tröster G. Sensor for Measuring Strain in Textile. Sensors. 2008;8(6):3719.

[11] Yamada T, Hayamizu Y, Yamamoto Y, Yomogida Y, Izadi-Najafabadi A, Futaba DN, et al. A stretchable carbon nanotube strain sensor for human-motion detection. Nat Nano. 2011;6(5):296-301.

[12] Kim KS, Zhao Y, Jang H, Lee SY, Kim JM, Kim KS, et al. Large-scale pattern growth of graphene films for stretchable transparent electrodes. Nature. 2009;457(7230):706-710. [13] Chen QZ, Harding SE, Ali NN, Lyon AR, Boccaccini AR. Biomaterials in cardiac tissue engineering: Ten years of research survey. Mat Sci Eng R. 2008;59(1-6):1-37.

[14] Costa P, Ferreira A, Sencadas V, Viana JC, Lanceros-Méndez S. Electro-mechanical properties of triblock copolymer styrene-butadiene-styrene/carbon nanotube composites for large deformation sensor applications. Sensors and Actuators A: Physical. 2013;201:458467.

[15] Kharissova OV, Kharisov BI, de Casas Ortiz EG. Dispersion of carbon nanotubes in water and non-aqueous solvents. RSC Advances. 2013;3(47):24812-24852.

[16] Byrne MT, Gun'ko YK. Recent Advances in Research on Carbon Nanotube-Polymer Composites. Advanced Materials. 2010;22(15):1672-1688.

[17] Wang Y, Ameer GA, Sheppard BJ, Langer R. A tough biodegradable elastomer. Nature biotechnology. 2002;20(6):602-606. 
[18] Rai R, Tallawi M, Grigore A, Boccaccini AR. Synthesis, properties and biomedical applications of poly(glycerol sebacate) (PGS): A review. Progress in Polymer Science. 2012;37(8):1051-1078.

[19] Chen QZ, Ishii H, Thouas GA, Lyon AR, Wright JS, Blaker JJ, et al. An elastomeric patch derived from poly(glycerol sebacate) for delivery of embryonic stem cells to the heart. Biomaterials. 2010;31(14):3885-3893.

[20] Gaharwar AK, Patel A, Dolatshahi-Pirouz A, Zhang H, Rangarajan K, Iviglia G, et al. Elastomeric nanocomposite scaffolds made from poly(glycerol sebacate) chemically crosslinked with carbon nanotubes. Biomaterials Science. 2015.

[21] Kang JH, Park C, Scholl JA, Brazin AH, Holloway NM, High JW, et al. Piezoresistive characteristics of single wall carbon nanotube/polyimide nanocomposites. Journal of Polymer Science Part B: Polymer Physics. 2009;47(10):994-1003.

[22] ASTM D790-15e2, Standard Test Methods for Flexural Properties of Unreinforced and Reinforced Plastics and Electrical Insulating Materials. vol. ASTM D790-15e2 West Conshohocken, PA: ASTM International; 2015.

[23] Sperling LH. Introduction to physical polymer science. New York: Wiley; 1992.

[24] Chen QZ, Bismarck A, Hansen U, Junaid S, Tran MQ, Harding SE, et al. Characterisation of a soft elastomer poly(glycerol sebacate) designed to match the mechanical properties of myocardial tissue. Biomaterials. 2008;29(1):47-57.

[25] Araujo J, Padrão J, Silva JP, Dourado F, Correia DM, Botelho G, et al. Processing and characterization of $\alpha$-elastin electrospun membranes. Applied Physics A. 2014;115(4):12911298.

[26] Makireddi S, S S, Kosuri G, Varghese FV, Balasubramaniam K. Electro-elastic and piezoresistive behavior of flexible MWCNT/PMMA nanocomposite films prepared by solvent 
casting method for structural health monitoring applications. Composites Science and Technology. 2015;118:101-107.

[27] Dawson JC, Adkins CJ. Conduction mechanisms in carbon-loaded composites. Journal of Physics: Condensed Matter. 1996;8(43):8321.

[28] Theodosiou TC, Saravanos DA. Numerical investigation of mechanisms affecting the piezoresistive properties of CNT-doped polymers using multi-scale models. Composites Science and Technology. 2010;70(9):1312-1320.

[29] Michelis F, Bodelot L, Bonnassieux Y, Lebental B. Highly reproducible, hysteresis-free, flexible strain sensors by inkjet printing of carbon nanotubes. Carbon. 2015;95:1020-1026. [30] Wang Y, Yang R, Shi Z, Zhang L, Shi D, Wang E, et al. Super-Elastic Graphene Ripples for Flexible Strain Sensors. ACS Nano. 2011;5(5):3645-3650. 

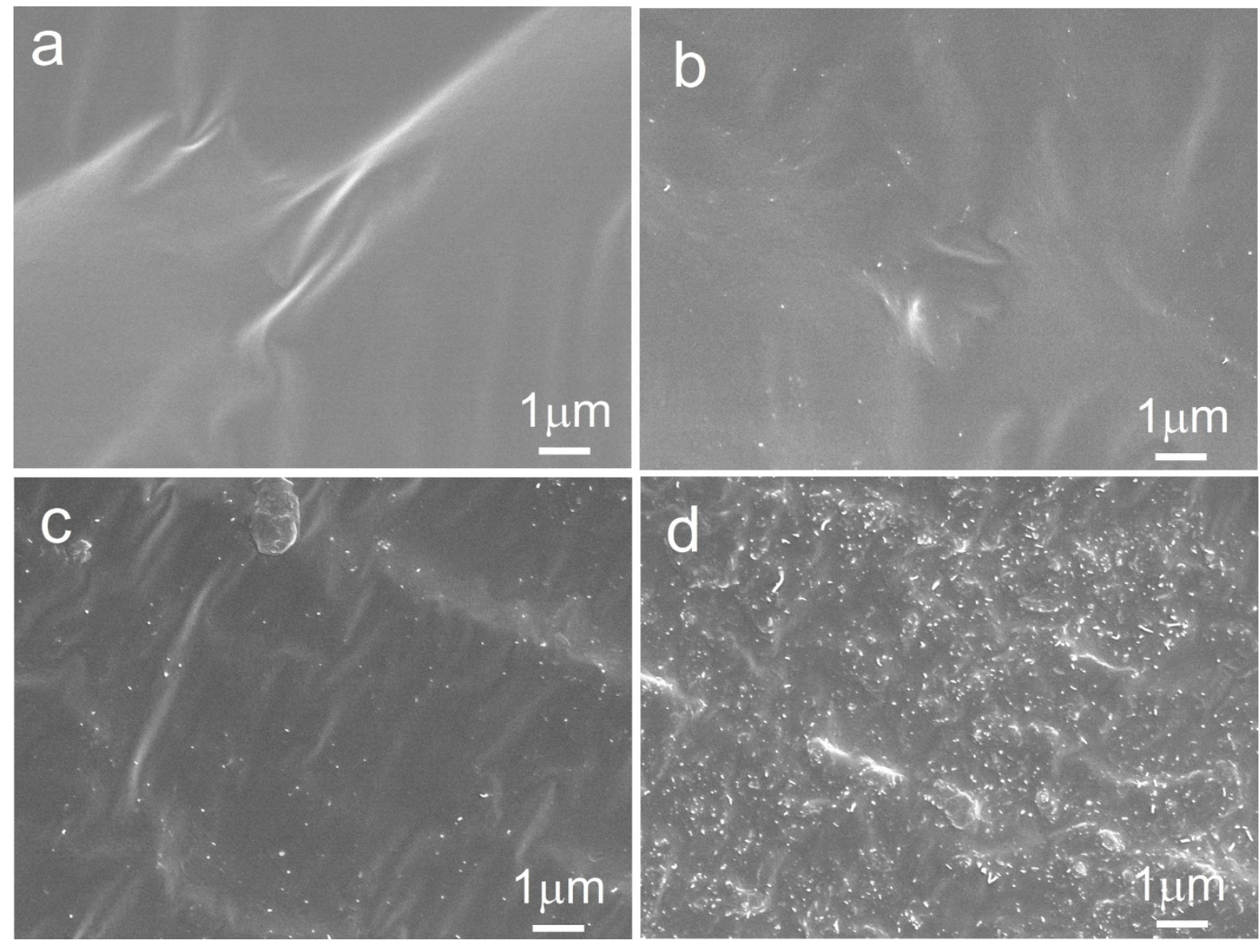

Figure 1 


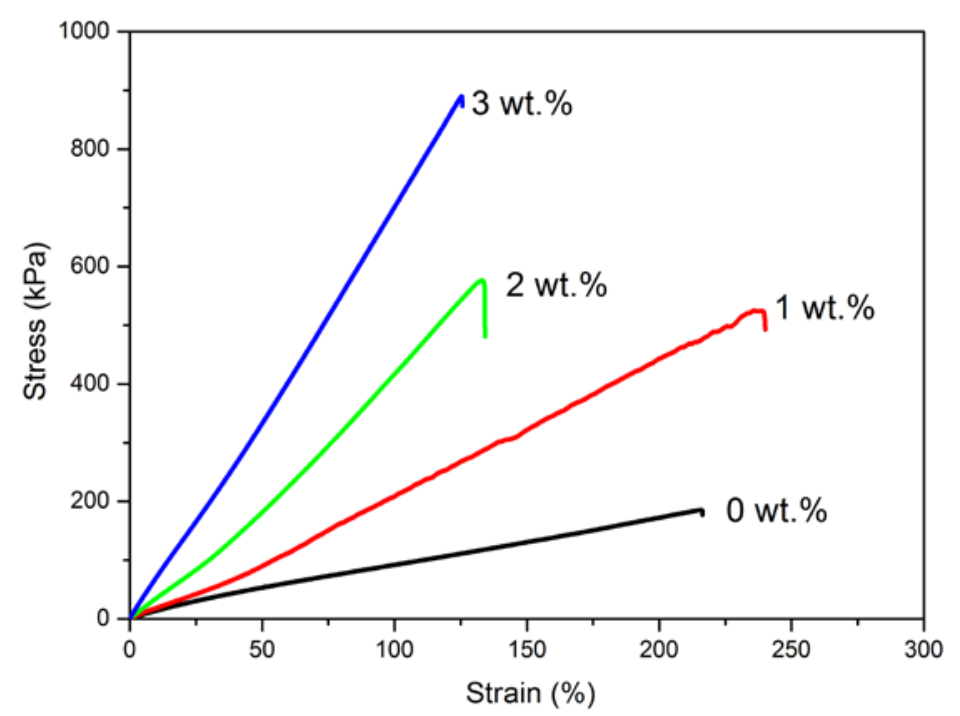

Figure 2

a

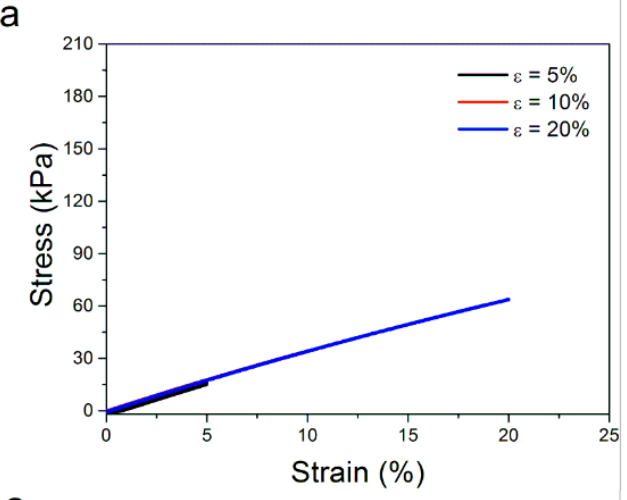

C

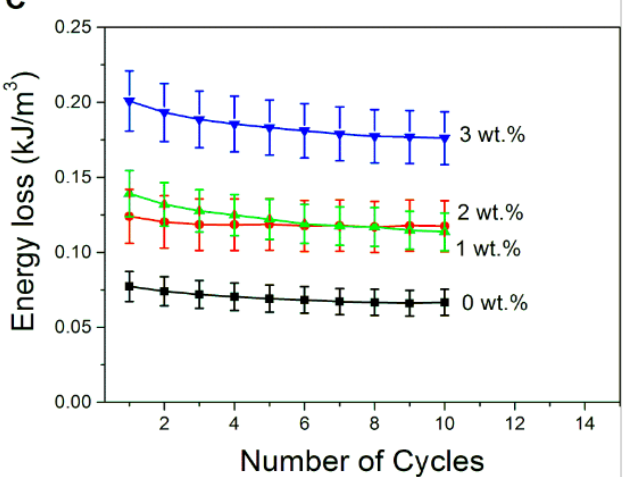

b

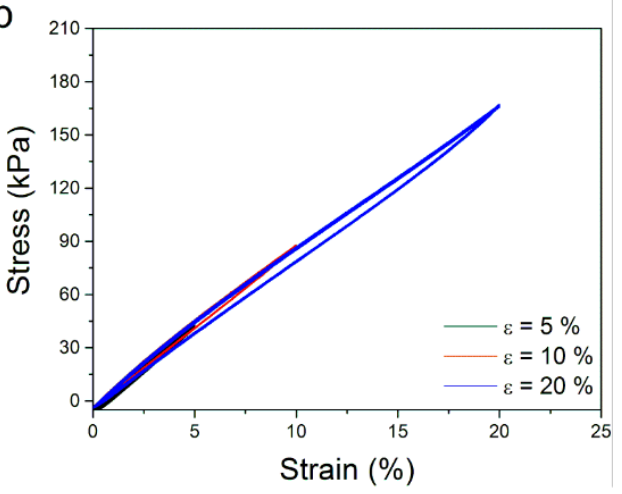

d

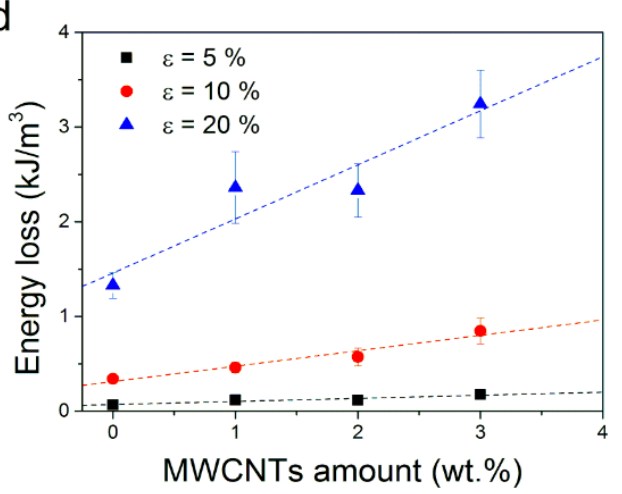

Figure 3 


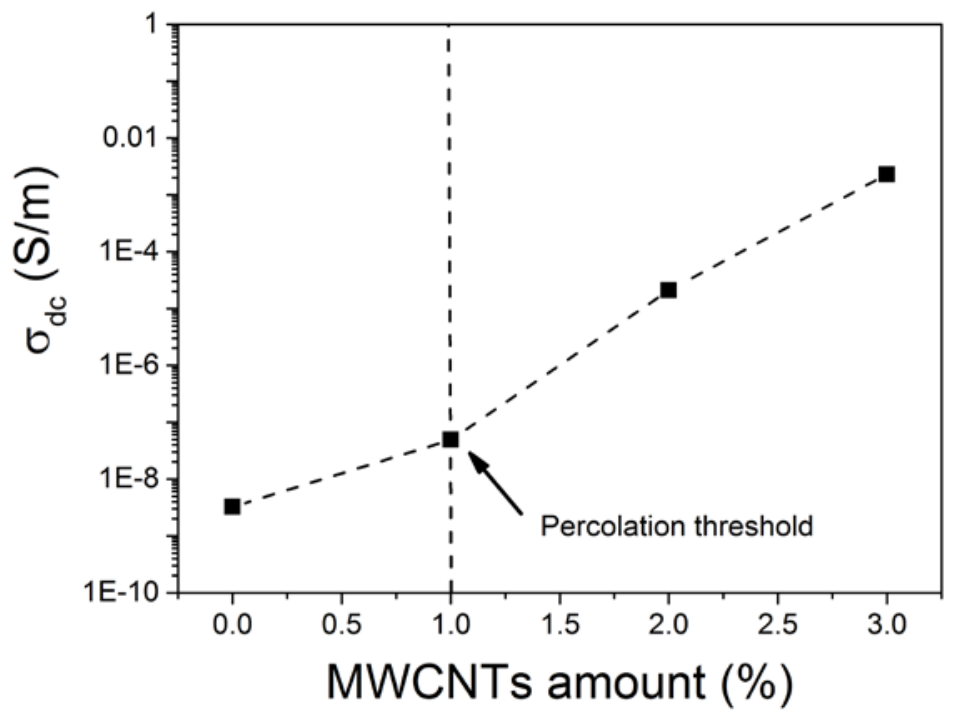

Figure 4
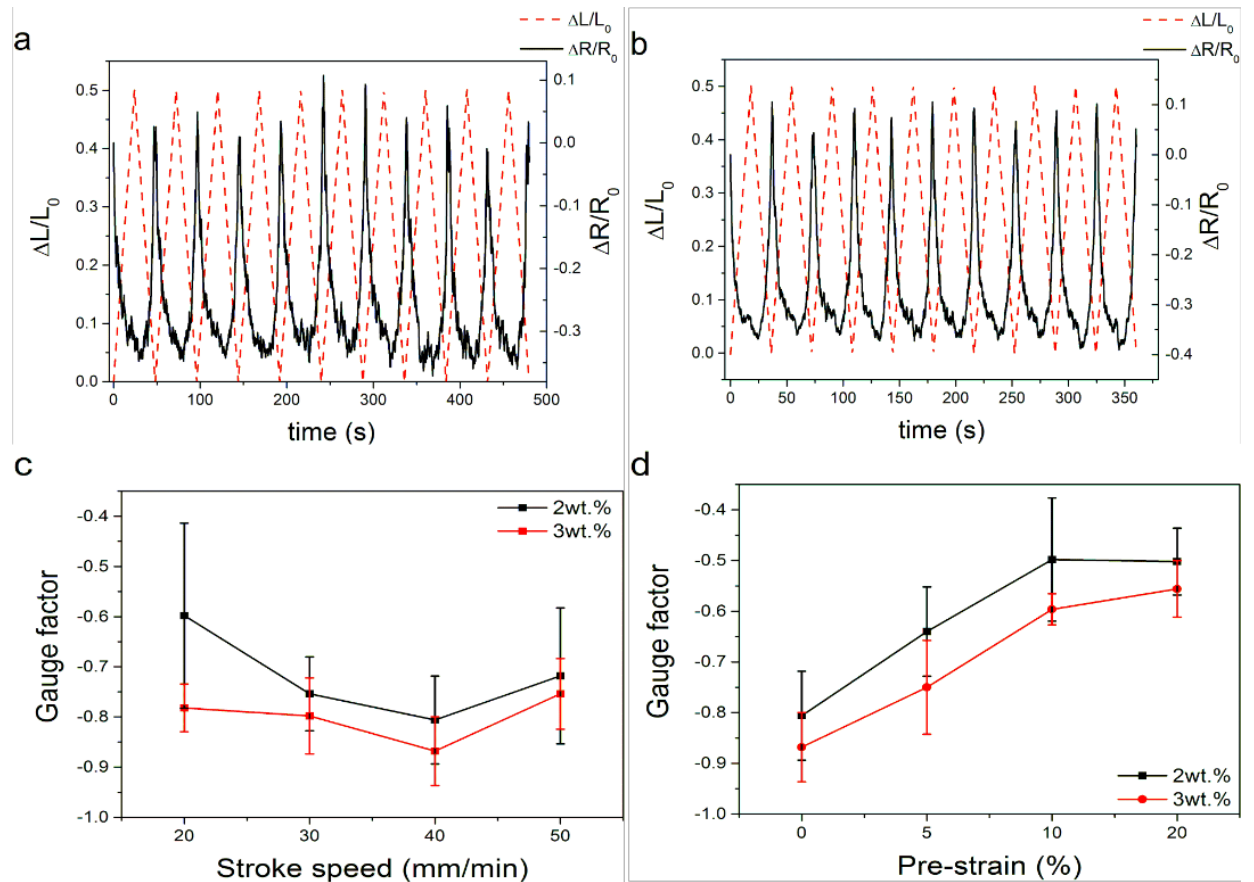

Figure 5 

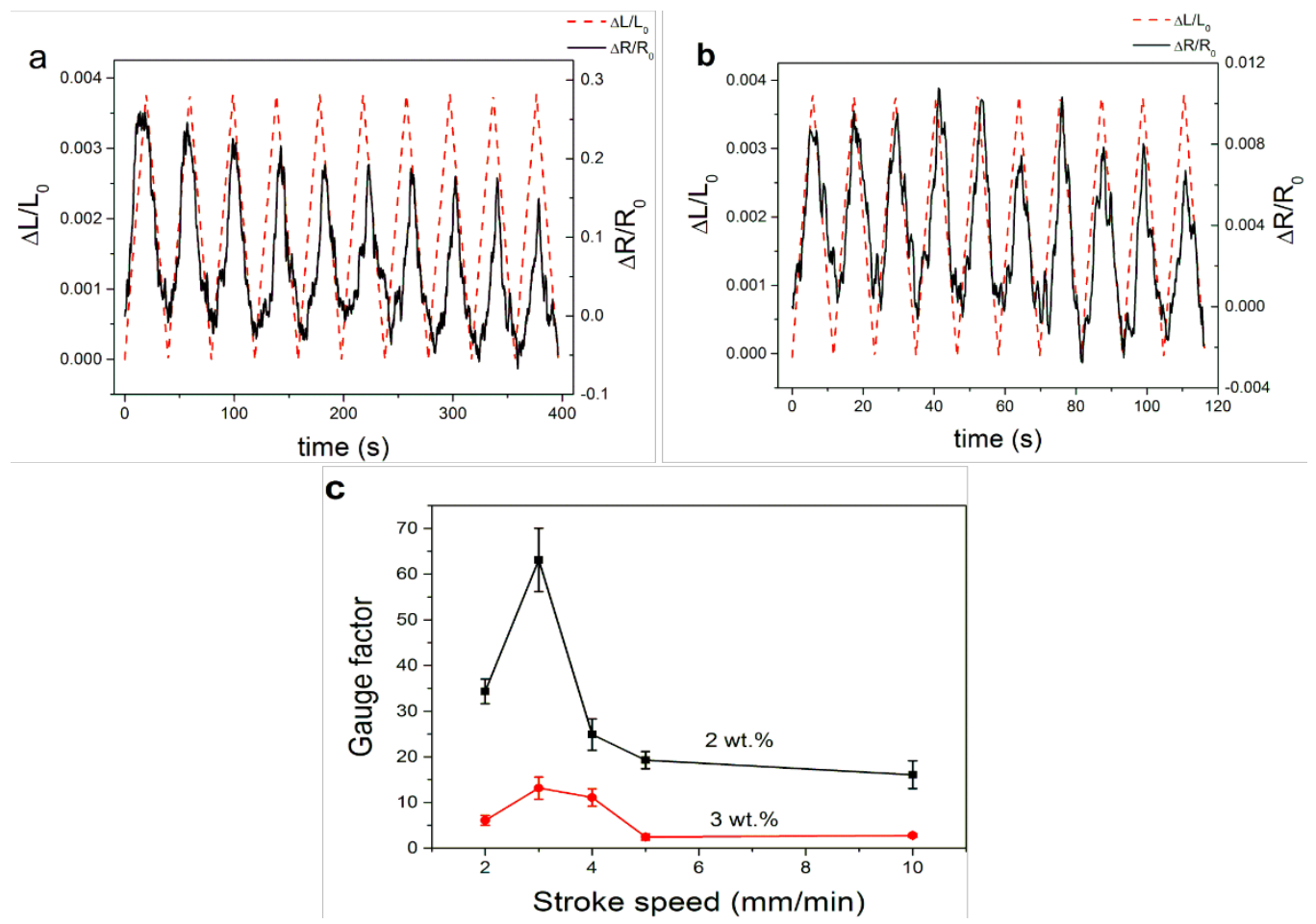

Figure 6

Table 1 -

\begin{tabular}{c|rccc}
\hline $\begin{array}{c}\text { MWCNT } \\
\text { amount }\end{array}$ & Ultimate & Ultimate stress & $\begin{array}{l}\text { Young's } \\
\text { strain (\%) }\end{array}$ & Crosslinking \\
(wt.\%) & & & & \\
\hline $\mathbf{0}$ & $219 \pm 8$ & $175 \pm 11$ & $88 \pm 9$ & $11 \pm 1$ \\
$\mathbf{1}$ & $233 \pm 25$ & $516 \pm 74$ & $216 \pm 13$ & $30 \pm 1$ \\
$\mathbf{2}$ & $134 \pm 8$ & $645 \pm 51$ & $434 \pm 39$ & $65 \pm 5$ \\
$\mathbf{3}$ & $119 \pm 13$ & $893 \pm 102$ & $764 \pm 44$ & $101 \pm 4$ \\
\hline
\end{tabular}

